\title{
Hot Dense Matter: Deconfinement and Clustering of Color Sources in Nuclear Collisions
}

\author{
Rolf P. Scharenberg ${ }^{1}$, Brijesh K. Srivastava ${ }^{1, *}$, Andrew S. Hirsch ${ }^{1}$ and Carlos Pajares ${ }^{2}$ \\ 1 Department of Physics and Astronomy, Purdue University, West Lafayette, IN 47907, USA; \\ schrnbrg@purdue.edu (R.P.S.); hirsch@purdue.edu (A.S.H.) \\ 2 Departamento de Fisica de Particulas, Universidale de Santiago de Compostela and Instituto Galego de \\ Fisica de Atlas Enerxias(IGFAE), 15782 Santiago de Compostela, Spain; pajares@fpaxp1.usc.es \\ * Correspondence: brijesh@purdue.edu
}

Received: 10 August 2018; Accepted: 10 September 2018; Published: 18 September 2018

check for updates

\begin{abstract}
Within the first few microseconds from after the Big Bang, the hot dense matter was in the form of the Quark Gluon Plasm (QGP) consisting of free quarks and gluons. By colliding heavy nuclei at RHIC and LHC at a velocity close to the speed of light, we were able to create the primordial matter and observe the matter after expansion and cooling. In this report we present the thermodynamics and transport coefficients obtained in the framework of clustering of color sources in both hadron-hadron and nucleus-nucleus collisions at RHIC and LHC energies. Multiparticle production at high energies can be described in terms of color strings stretched between the projectile and target. At high string density single strings overlap and form color sources. This addition belongs to the non-perturbative domain of Quantum Chromo Dynamics (QGP) and manifests its most fundamental features. The Schwinger $\mathrm{QED}_{2}$ mechanism produces color neutral $q \bar{q}$ pairs when color source strings break. Subsequent hardonization produces the observed hadrons. With growing energy and atomic number of the colliding nuclei the density of strings grows and more color sources form clusters in the transverse plane. At a certain critical density a macroscopic cluster appears, which marks the percolation phase transition. This is the Color String Percolation Model (CSPM). The critical density is identified as the deconfinement transition and happens at the hadronization temperature. The stochastic thermalization in $p p$ and $\mathrm{A}-\mathrm{A}$ is a consequence of the quantum tunneling through the event horizon introduced by the confining color fields, the Hawking-Unruh effect. The percolation approach within CSPM is successfully used to describe the crossover phase transition in the soft collision region. The same phenomenology when applied to both hadron-hadron and nucleus-nucleus collisions emphasizes the importance of color string density, creating a macroscopic cluster which identifies the connectivity required for a finite droplet of the QGP.
\end{abstract}

Keywords: QGP; deconfinement

\section{Introduction}

What is the behavior of matter when we increase its density? The observed densities of our world expand over many orders of magnitude, from $\sim 10^{-6}$ nucleons $/ \mathrm{cm}^{3}$ on average in our universe to $\sim 10^{38}$ nucleons $/ \mathrm{cm}^{3}$ inside a nucleus and $10^{39}$ nucleons $/ \mathrm{cm}^{3}$ in a neutron star. The study of the high density limit, specifically the confinement transition from hadrons to quarks and gluons can be regarded as the place where a high energy collision (two body) probes the short range limit and therefore meets the thermodynamics (many body) of this short distance dynamics.

In the case of Quantum-Chromo-Dynamics (QCD) we have confinement, which means the color field is confined to a small space region, forming hadrons which are colorless bound states of colored quarks. In the confining phase the quarks are dressed with gluons, acquiring an effective constituent 
mass up to $300 \mathrm{MeV}$ (1/3 of the proton mass). For light quarks the constituent mass is much larger than the mass appearing in the QCD Lagrangian which is close to zero. In other words the light quark mass in the confined phase is generated by the confinement interaction and when deconfinement occurs the additional mass disappears.

The lattice QCD studies have shown, that at baryon chemical density $\mu_{B} \sim 0$, color deconfinement and chiral symmetry restoration coincide. Hence, in a medium of low baryon density, the mass of the constituent quark vanishes at the deconfinement point $T_{\mathcal{C}}$ and also the screening radius of the gluon cloud size vanishes. At low $T$ and high $\mu$, there is no reason to expect a similar behavior and probably there may be an intermediate region of massive dressed quarks between the hadronic phase and the deconfined and chiral symmetry restoration phase. At low $T$ and high $\mu$, other possibilities could exist such as quarkyonic matter and color superconductivity [1,2]. A possible phase diagram is shown in Figure 1 [3].

The energy density of quark-gluon matter at a temperature $T$ is given by

$$
\epsilon=\frac{\pi^{2}}{30}\left[g_{g}+\frac{7}{8}\left(g_{q}+g_{\bar{q}}\right)\right] T^{4}
$$

where $g_{g}, g_{q}$ and $g_{\bar{q}}$ are the degeneracy numbers of the gluons, quarks and antiquarks. For two flavors the total number of degrees of freedom $(\mathrm{DOF}) \sim 37$.

Quantitative predictions have not been evaluated using experimental data from high energy heavy ion collisions. In one of the recent article several big questions regarding the formation of QGP have been posed [4]. The two most interesting problems are the determination of temperature and the thermalization in $p p$ and $A A$ collisions. The central challenge of this paper is to obtain the thermodynamics of the QGP from data and compare with the predictions of lattice QCD results. There are other review articles in the literature on the deconfinement both in $p p$ and $A A$ collisions [5-7].

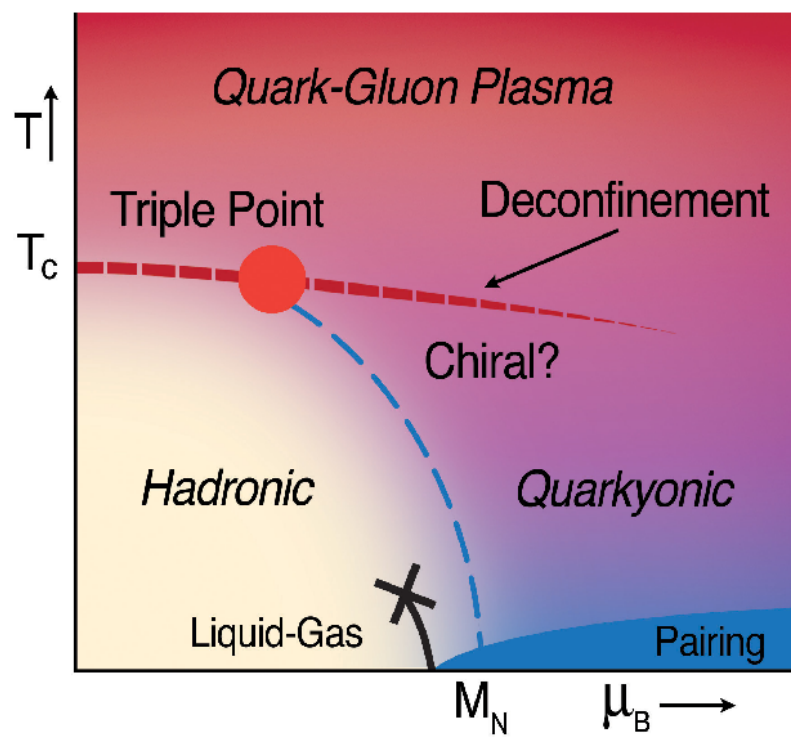

Figure 1. Phase diagram of the nuclear matter. Temperature vs. baryonic chemical potential [3].

\section{High Energy Nuclear Collisions}

A diagram of the time sequence of a heavy ion collision is shown in Figure 2. The time unit is $\mathrm{fm} / \mathrm{c}=3.3 \times 10^{-24} \mathrm{~s}$. The initial energy density is developed in $1 \mathrm{fm} / \mathrm{c}$. A new form of matter, the QGP, is composed of a dense volume of deconfined $Q$ and $\bar{Q}$ pairs, which expands in $\sim 10 \mathrm{fm} / \mathrm{c}$ and subsequently forms the freeze out hadrons. 


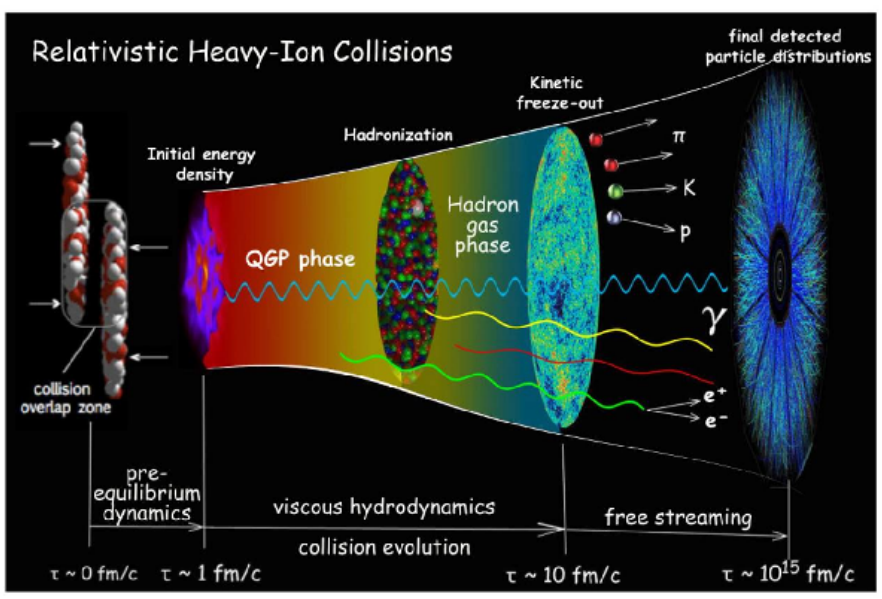

Figure 2. Illustration of the dynamical evolution of relativistic heavy-ion collisions and the QGP expansion [8].

To create a system of a large number $(\sim 1000)$ of color strings, in a nuclear collision requires an accelerator with $\sim 100 \times 10^{9} \mathrm{eV}(100 \mathrm{GeV})$ kinetic energy per nucleon with two colliding beams. The two accelerators used in these nuclear collision studies are the Relativistic Heavy Ion Collider (RHIC) located at Brookhaven National Laboratory on Long Island New York and the Large Hadron Collider (LHC) at the Central European Research Nuclear Laboratory (CERN), in Geneva, Switzerland.

The strong field of a color source can lift a quark-antiquark pair out of the vacuum. This is the Schwinger $Q E D_{2}$ mechanism [9]. The interaction of an initial quark $Q$ in one nucleon and $\bar{Q}$ in the colliding nucleon are connected by the strong color string that subsequently breaks into two strings creating a $q$ and $\bar{q}$ deconfined pair at a time $\tau_{\text {pro }}$ given by [9]

$$
\tau_{\text {pro }}=\frac{2.405 \hbar}{\left\langle m_{t}\right\rangle} \approx 1 \mathrm{fm} / \mathrm{c} .
$$

with $\left\langle m_{t}\right\rangle$ being the average transverse mass of the $q \bar{q}$ pair. More details of string fragmentation is given by Lund model which has tried to address the collectivity in $p p$ collisions [10].

\section{The Color String Percolation Model}

The thermodynamic quantities are derived from RHIC and LHC data using the Color String Percolation Model (CSPM), as seen in Figure 3. In CSPM strings have a finite area in the transverse space in terms of the color field. The area is restricted to a finite disc of a given radius dictated by confinement. When the string with radii $r_{0}$ and string density $\xi$ are uniformly distributed on the much larger transverse nuclear collision area $S_{N}$ they may overlap. $\xi$ is given by $N_{s} S_{1} / S_{N}$ where the single disc area is $S_{1}$ and $N_{s}$ is the total number of strings.

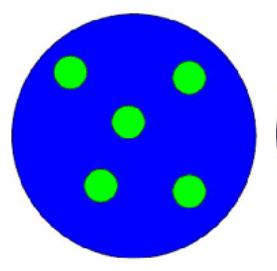

Isolated Disks

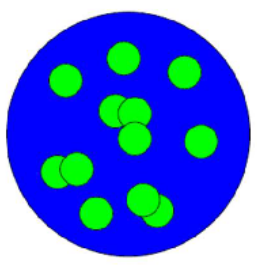

Clusters

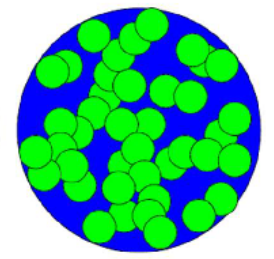

Percolation

Figure 3. (Left) Isolated disks; (Middle) Cluster formation; (Right) Overlapping discs forming a cluster of communication [11]. 
Multiparticle production is currently described in terms of color strings stretched between the projectile and the target, which decay into new strings and subsequently hadronize to produce the observed hadrons. Color strings may be viewed as small areas in the transverse plane filled with color field created by colliding partons. With growing energy and size of the colliding system, the number of strings grows, and they start to overlap, forming clusters, in the transverse plane. At a certain critical density $\xi_{c}=1.2$ a macroscopic percolation cluster appears that marks the percolation phase transition [11-13]. This is the Color String Percolation Model (CSPM). The interaction between strings occurs when they overlap and the general result, due to the $\mathrm{SU}(3)$ random summation of charges, is a reduction in multiplicity and an increase in the string tension hence increase in the average transverse momentum squared, $\left\langle p_{t}^{2}\right\rangle$. We assume that a cluster of $n$ strings that occupies an area of $S_{n}$ behaves as a single color source with a higher color field $\vec{Q}_{n}$ corresponding to the vectorial sum of the color charges of each individual string $\overrightarrow{Q_{1}}$. The resulting color field covers the area of the cluster. As $\overrightarrow{Q_{n}}=\sum_{1}^{n}{\overrightarrow{Q_{1}}}_{1}$, and the individual string colors may be oriented in an arbitrary manner respective to each other, the average $\overrightarrow{Q_{1 i}} \overrightarrow{Q_{1 j}}$ is zero, and $\overrightarrow{Q_{n}^{2}}=n \overrightarrow{Q_{1}^{2}}$.

Knowing the color charge $\vec{Q}_{n}$ one can obtain the multiplicity $\mu_{n}$ and the mean transverse momentum squared $\left\langle p_{t}^{2}\right\rangle_{n}$ of the particles produced by a cluster of $n$ strings [13]

$$
\mu_{n}=\sqrt{\frac{n S_{n}}{S_{1}}} \mu_{1} ; \quad\left\langle p_{t}^{2}\right\rangle_{n}=\sqrt{\frac{n S_{1}}{S_{n}}}\left\langle p_{t}^{2}\right\rangle_{1}
$$

where $\mu_{1}$ and $\left\langle p_{t}^{2}\right\rangle_{1}$ are the mean multiplicity and $\left\langle p_{t}^{2}\right\rangle$ of particles produced from a single string with a transverse area $S_{1}=\pi r_{0}^{2}$. For strings just touching each other $S_{n}=n S_{1}$, and $\mu_{n}=n \mu_{1},\left\langle p_{t}^{2}\right\rangle_{n}=\left\langle p_{t}^{2}\right\rangle_{1}$. When color strings fully overlap, $S_{n}=S_{1}$ and therefore $\mu_{n}=\sqrt{n} \mu_{1}$ and $\left\langle p_{t}^{2}\right\rangle_{n}=\sqrt{n}\left\langle p_{t}^{2}\right\rangle_{1}$, so that the multiplicity is maximally suppressed and the $\left\langle p_{t}^{2}\right\rangle_{n}$ is maximally enhanced. This implies a simple relation between the multiplicity and transverse momentum $\mu_{n}\left\langle p_{t}^{2}\right\rangle_{n}=n \mu_{0}\left\langle p_{t}^{2}\right\rangle_{1}$, which is conservation of the total transverse momentum.

In the thermodynamic limit, one obtains an analytic expression $[12,13]$

$$
\left\langle\frac{n S_{1}}{S_{n}}\right\rangle=\frac{\xi}{1-e^{-\xi}} \equiv \frac{1}{F(\xi)^{2}}
$$

where $(F \xi)$ is the color suppression factor. With $F(\xi) \rightarrow 1$ as $\xi \rightarrow 0$ and $F(\xi) \rightarrow 0$ as $\xi \rightarrow \infty$, where $\xi=\frac{N_{S} S_{1}}{S_{N}}$ is the percolation density parameter. Equation(3) can be written as $\mu_{n}=n F(\xi) \mu_{0}$ and $\left\langle p_{t}^{2}\right\rangle_{n}=\left\langle p_{t}^{2}\right\rangle_{1} / F(\xi)$. The critical cluster which spans $S_{N}$, appears for $\xi_{c} \geq 1.2$ [14].

It is worth noting that CSPM is a saturation model similar to the Color Glass Condensate (CGC), where $\left\langle p_{t}^{2}\right\rangle_{1} / F(\xi)$ plays the same role as the saturation momentum scale $Q_{s}^{2}$ in the CGC model $[15,16]$. Saturation results from the overcrowding in impact parameter of low $x$ partons of a boosted hadrons or nucleus, leading to the appearance of a scale, $Q_{s}^{2}$. This is the basic idea of CGC. For example the particle density in CSPM is given by

$$
\frac{d n}{d y} \sim\left(1-e^{-\tau}\right)^{1 / 2} N_{\text {part }}
$$

In CGC particle density is related to the coupling constant $\alpha_{s}\left(Q_{s}^{2}\right)$,

$$
\frac{d n}{d y} \sim \frac{1}{\alpha_{s}\left(Q_{s}^{2}\right)} N_{\text {part }}
$$

In both case particle density increases with the number of participants $N_{\text {part }}$ [16]. 


\section{Color Suppression Factor}

In the present work we have extracted the color suppression factor $F(\xi)$ in high multiplicity events in $p p$ collisions using CMS data from the transverse momentum spectra of pions at $\sqrt{s}=0.9$, $2.76,7$ and $13 \mathrm{TeV}[17,18]$. Figure 4 shows a transverse momentum spectra for two multiplicity cuts at $\sqrt{s}=7 \mathrm{TeV}$. For comparison purpose the spectra from $p p$ collisions $\sqrt{s}=200 \mathrm{GeV}$ is also shown. The spectra becomes harder for higher multiplicity cuts. This is due to the fact that high string density color sources are created in the higher multiplicity events.

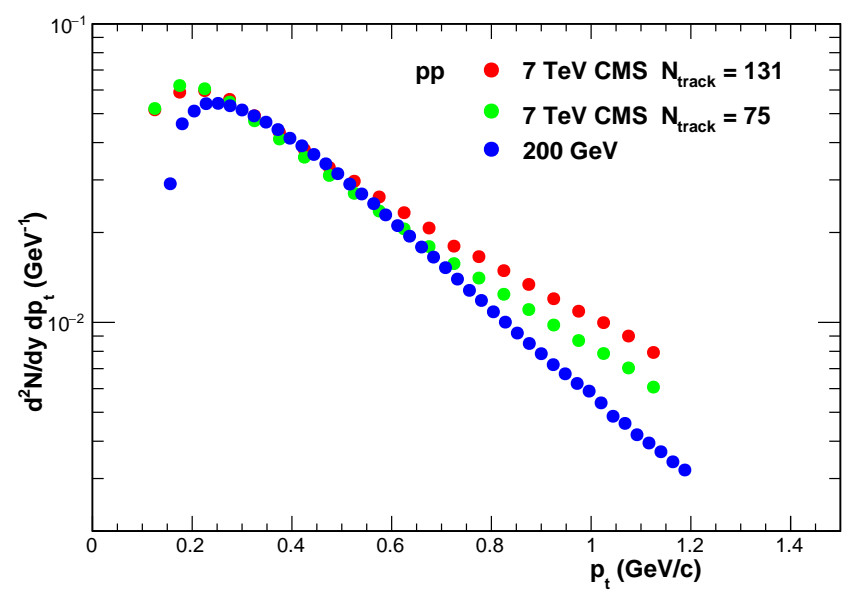

Figure 4. Transverse momentum spectra of pions from CMS experiment at $\sqrt{s}=7 \mathrm{TeV}$ for two different multiplicity cuts $N_{\text {track }}=131$ (red solid circle) and $N_{\text {track }}=75$ (green solid circle) [17]. For comparison purpose the $p_{t}$ spectra from $p p$ at $\sqrt{s}=200 \mathrm{GeV}$ is also shown (solid blue circle) [19].

To evaluate the initial value of $F(\xi)$ from data in high multiplicity events in $p p$ collisions, a parameterization of the experimental data of $p_{t}$ distribution in low energy $p p$ collisions $\sqrt{s}=200 \mathrm{GeV}$ was used [20]. The charged particle spectrum is described by a power law [19]

$$
d^{2} N_{c} / d p_{t}^{2}=a /\left(p_{0}+p_{t}\right)^{\alpha},
$$

where $a$ is the normalization factor, $p_{0}$ and $\alpha$ are fitting parameters with $p_{0}=1.98$ and $\alpha=12.87$ [20]. This parameterization is used in high multiplicity events in $p p$ collisions to take into account the interactions of the strings [19]. The color suppression factor $F(\xi)$ encodes the effect of the interaction among strings once they overlap. The parameter $p_{0}$ in Equation (7) is for independent strings and gets modified.

$$
\begin{aligned}
p_{0} & \rightarrow p_{0}\left(\frac{\left\langle n S_{1} / S_{n}\right\rangle_{p p}^{m u l t}}{\left\langle n S_{1} / S_{n}\right\rangle_{p p}}\right)^{1 / 4}, \\
\frac{d^{2} N_{c}}{d p_{T}^{2}}= & \frac{a}{\left(p_{0} \sqrt{F(\xi)_{p p} / F(\xi)_{p p}^{m u l t}}+p_{T}\right)^{\alpha}} .
\end{aligned}
$$

where $F(\xi)_{p p}^{m u l t}$ is the multiplicity dependent color suppression factor. In $p p$ collisions $F(\xi)_{p p} \sim 1$ at low energies due to the low overlap probability. The spectra were fitted using Equation (9) in the softer sector with $p_{t}$ in the range $0.12-1.0 \mathrm{GeV} / \mathrm{c}$. Equation (9) is similar to Tsallis distribution [21] which can be obtained in the clustering of color sources frame by doing the convolution of the distribution of the different formed clusters size with the thermal distributions of the decay of each 
cluster. In the thermodynamic limit the color suppression factor $F(\xi)$ is related to the percolation density parameter $\xi[19]$.

$$
F(\xi)=\sqrt{\frac{1-e^{-\xi}}{\xi}} .
$$

Figure 5 shows the extracted value of $F(\xi)$ as a function of $N_{\text {tracks }}$ from CMS experiment for $\sqrt{s}=0.9-13 \mathrm{TeV}$. It is observed that the $F(\xi)$ has a lower value for high multiplicity events. To compare high energy $p p$ with the heavy ions results, we need to normalized $N_{\text {tracks }}$ with the interaction area in $p p$ collisions.

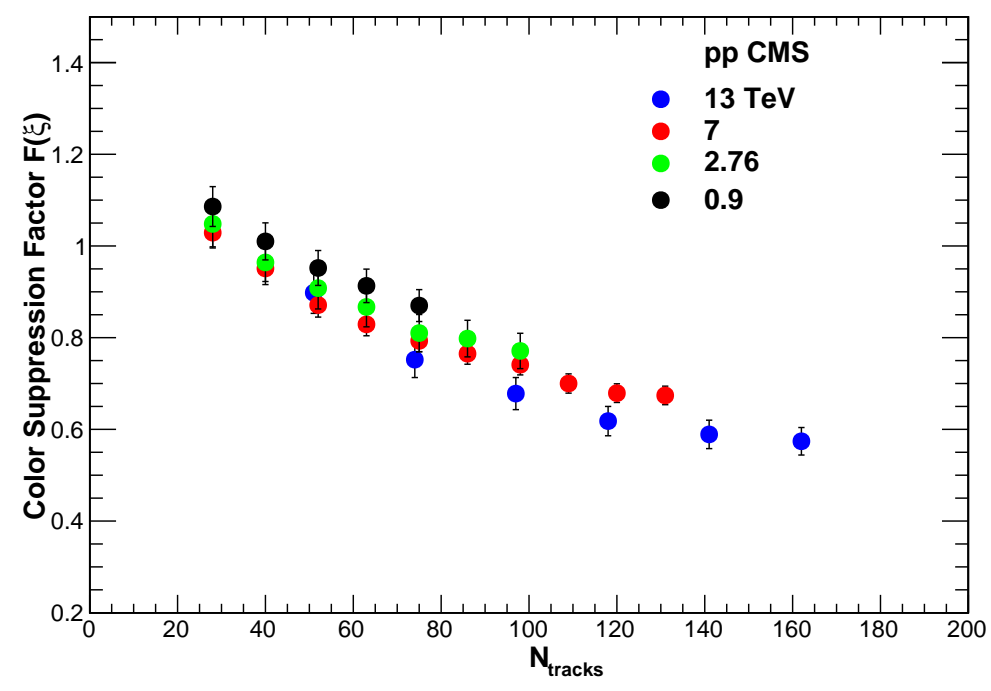

Figure 5. Color Suppression Factor $F(\xi)$ in $p p$ collisions vs. $N_{\text {tracks }}$.

The interaction area $S_{\perp}=\pi R_{p p}^{2}$ has been computed in the IP-Glasma model, where $R_{p p}$ is the interaction radius [22]. This is based on an impact parameter description of $p p$ collisions, combined with an underlying description of particle production based on the theory of Color Glass Condensate [22]. The interaction radius $R_{p p}$ is approximately a linear function of the charged particle multiplicity. In the IP-Glasma model $R_{p p}$ is dependent on gluon multiplicity [22]

$$
\begin{gathered}
R_{p p}=f_{p p}\left(d N_{g} / d y\right)^{1 / 3}, \\
f_{p p}= \begin{cases}0.387+0.0335 x+0.274 x^{2}-0.0542 x^{3}, & \text { if } x<3.4, \\
1.538 & \text { if } x \geq 3.4 .\end{cases}
\end{gathered}
$$

where $x=\left(d N_{g} / d y\right)^{1 / 3}$. The gluon multiplicity $d N_{g} / d y$ is related to the number of tracks seen in the CMS experiment by

$$
d N_{g} / d y \approx(3 / 2) \frac{1}{\Delta \eta} N_{\text {track }}
$$

where $\Delta \eta \sim 4.8$ units of pseudorapidity. The interaction cross section $S_{\perp}$ as a function of $N_{\text {tracks }} / \Delta \eta$, using Equation (11), is shown in Figure 6. $S_{\perp}$ increases with the multiplicity and for very high multiplicities it is approximately constant.

Figure 7 shows the extracted value of $F(\xi)$ as a function of $N_{\text {tracks }} / \Delta \eta$ scaled by the interaction area $S_{\perp}$ from the CMS experiment for $\sqrt{s}=0.9-13 \mathrm{TeV}$. $N_{\text {tracks }}$ is the total charged particle multiplicity in the region $|\eta|<2.4$ with $\Delta \eta \sim 4.8$ units of pseudorapidity. 


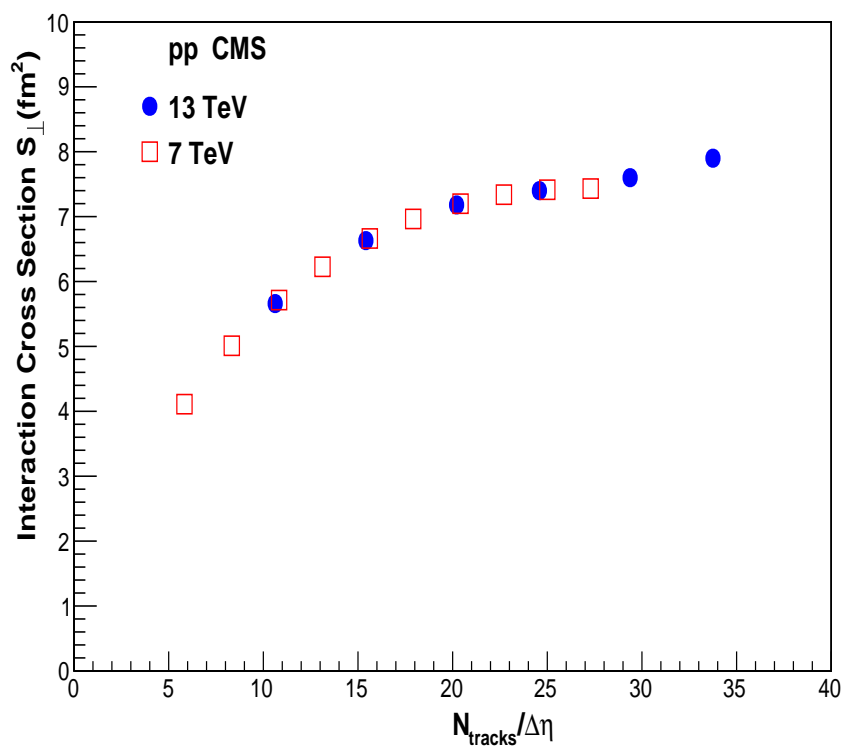

Figure 6. Interaction cross section $S_{\perp}$ vs. $N_{\text {tracks }} / \Delta \eta$. $S_{\perp}$ is obtained using IP-Glasma model [22].

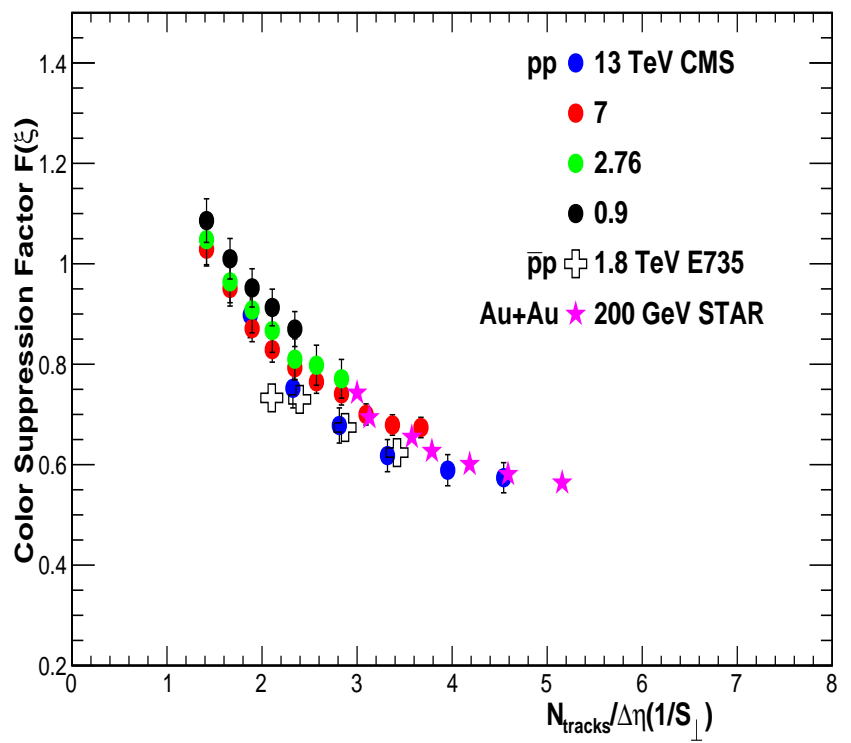

Figure 7. Color Suppression Factor $F(\xi)$ in $p p, \bar{p} p$ and Au-Au collisions vs. $N_{\text {tracks }} / \Delta \eta$ scaled by the transverse area $S_{\perp}$. For $p p$ and $\bar{p} p$ collisions $S_{\perp}$ is multiplicity dependent as obtained from IP-Glasma model [22]. In case of Au-Au collisions the nuclear overlap area was obtained using the Glauber model [23].

The results from FNAL (Fermi National Accelerator Laboratory) E735 experiment on $\bar{p} p$ collisions at $\sqrt{s}=1.8 \mathrm{TeV}$ is also shown in Figure 7 [24]. In the E735 experiment the total charged particle multiplicity was $10<N_{\text {tracks }}<200$ in the pseudorapidity range $|\eta|<3.25$ with $\Delta \eta \sim 6.5$ units of pseudorapidity. It is observed that the E735 results follow the trend seen in CMS data. The decrease in $F(\xi)$ for high multiplicity events is due to the high string density created in theses events.

\section{Temperature}

The connection between $F(\xi)$ and the temperature $T(\xi)$ involves the Schwinger mechanism (SM) for particle production. The Schwinger distribution for massless particles is expressed in terms of $p_{t}^{2}[9,25]$ 


$$
d n / d p_{t}^{2} \sim \exp \left(-\pi p_{t}^{2} / x^{2}\right)
$$

where the average value of the string tension is $\left\langle x^{2}\right\rangle$. The tension of the macroscopic cluster fluctuates around its mean value because the chromo-electric field is not constant. The origin of the string fluctuation is related to the stochastic picture of the QCD vacuum. Since the average value of the color field strength must vanish, it cannot be constant but changes randomly from point to point $[26,27]$. Such fluctuations lead to a Gaussian distribution of the string tension

$$
\frac{d n}{d p_{t}^{2}} \sim \sqrt{\frac{2}{<x^{2}>}} \int_{0}^{\infty} d x \exp \left(-\frac{x^{2}}{2<x^{2}>}\right) \exp \left(-\pi \frac{p_{t}^{2}}{x^{2}}\right)
$$

which gives rise to thermal distribution [26]

$$
\frac{d n}{d p_{t}^{2}} \sim \exp \left(-p_{t} \sqrt{\frac{2 \pi}{\left\langle x^{2}\right\rangle}}\right)
$$

with $\left\langle x^{2}\right\rangle=\pi\left\langle p_{t}^{2}\right\rangle_{1} / F(\xi)$. The temperature is expressed as [20,28]

$$
T(\xi)=\sqrt{\frac{\left\langle p_{t}^{2}\right\rangle_{1}}{2 F(\xi)}}
$$

We will adopt the point of view that the experimentally determined chemical freeze-out temperature is a good measure of the upper end of the cross over phase transition temperature, $T_{\mathcal{C}}$ [29]. The single string average transverse momentum $\left\langle p_{t}^{2}\right\rangle_{1}$ is calculated at $\xi_{c}=1.2$ with the universal chemical freeze-out temperature of $167.7 \pm 2.6 \mathrm{MeV}$ [30]. This gives $\sqrt{\left\langle p_{t}^{2}\right\rangle_{1}}=207.2 \pm 3.3 \mathrm{MeV}$ which is close to $\simeq 200 \mathrm{MeV}$ used previously in the calculation of percolation transition temperature [28].

Recently, it has been suggested that fast thermalization in heavy ion collisions can occur through the existence of an event horizon caused by a rapid deceleration of the colliding nuclei [31]. The thermalization in this case is due to the Hawking-Unruh effect [32,33].

It is well known that the black holes evaporates by quantum pair production and behave as if they have an effective temperature of

$$
T_{H}=\frac{1}{8 \pi \mathrm{GM}^{\prime}}
$$

where $1 / 4 \mathrm{GM}$ is the acceleration of gravity at the surface of a black hole of mass $M$. The rate of pair production in the gravitational background of the black hole can be evaluated by considering the tunneling through the event horizon. Unruh showed that a similar effect arises in a uniformly accelerated frame, where an observer detects the thermal radiation with the temperature $T=a / 2 \pi$, where $a$ is the acceleration. Similarly, in hadronic interactions the probability to produce states of masses $M$ due to the chromoelectric field $E$ and color charge is given by the Schwinger mechanism [9]

$$
W_{M} \sim \exp \left(\frac{-\pi M^{2}}{g E}\right) \sim \exp (-M / T)
$$

which is similar to the Boltzmann weight in a heat bath with an effective temperature

$$
T=\frac{a}{2 \pi}, a=\frac{2 g E}{M} .
$$

In CSPM the strong color field inside the large cluster produces deceleration of the primary $q \bar{q}$ pair which can be seen as a thermal temperature by means of the Hawking-Unruh effect. This implies that the radiation temperature is determined by the transverse extension of the color flux tube/cluster in terms of the string tension [34-36]. 


$$
T=\sqrt{\frac{\sigma}{2 \pi}}
$$

The string percolation density parameter $\xi$ which characterizes the percolation clusters measures the initial temperature of the system. Since the cluster covers most of the interaction area, this temperature becomes a global temperature determined by the string density. In this way at $\xi_{c}=1.2$ the connectivity percolation transition at $T\left(\xi_{c}\right)$ models the thermal deconfinement transition. The temperature obtained using Equation (16) was $~ 193.6 \mathrm{MeV}$ for Au-Au collisions at $\sqrt{s_{N N}}=200 \mathrm{GeV}$ in reasonable agreement with $T_{i}=221 \pm 19^{\text {stat }} \pm 19^{\text {sys }} \mathrm{MeV}$ from the enhanced direct photon experiment measured by the PHENIX Collaboration [37]. For $\mathrm{Pb}-\mathrm{Pb}$ collisions at $\sqrt{s_{N N}}=2.76 \mathrm{TeV}$ the temperature is $\sim 262.2 \mathrm{MeV}$ for $0-5 \%$ centrality, which is expected to be $\sim 35 \%$ higher than the temperature from $\mathrm{Au}-\mathrm{Au}$ collisions [20]. A recent summary of the results from $\mathrm{Pb}-\mathrm{Pb}$ collisions at the LHC has mentioned that the initial temperature increases at least by $30 \%$ as compared to the top RHIC energy [38]. The direct photon measurements from ALICE gives the temperature of $T_{i}=304 \pm 51 \mathrm{MeV}$ [39]. The agreement with the measured temperature shows that the temperature obtained using Equation (16) can be termed as the initial temperature of the percolation cluster.

Figure 8 shows a plot of temperature as a function of $N_{\text {tracks }} / \Delta \eta$ scaled by $S_{\perp}$. Temperature from both hadron-hadron and nucleus-nucleus collisions fall on a universal curve when multiplicity is scaled by the transverse interaction area. The horizontal line at $\sim 165 \mathrm{MeV}$ is the universal hadronization temperature obtained from the systematic comparison of the statistical thermal model parametrization of hadron abundances measured in high energy $e^{+} e^{-}, p p$ and A-A collisions [30]. In Figure 8 for $\sqrt{s}=7$ and $13 \mathrm{TeV}$ higher multiplicity cuts show temperatures above the hadronization temperature and similar to those observed in Au-Au collisions at $\sqrt{s}_{N N}=200 \mathrm{GeV}$.

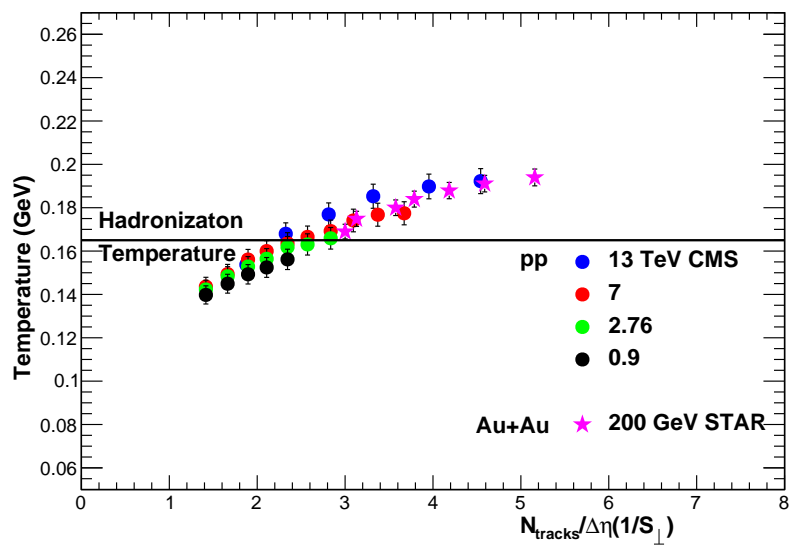

Figure 8. Temperature vs. $N_{\text {tracks }} / \Delta \eta$ scaled by $S_{\perp}$ from $p p$ and Au-Au collisions. The horizontal line at $\sim 165 \mathrm{MeV}$ is the universal hadronization temperature $[30,40]$.

The thermalization in $p p$ and A-A collisions occurs through the existence of an event horizon caused by a rapid deceleration of the colliding nuclei [34,35]. The thermalization is due to the Hawking-Unruh effect [32-34]. In CSPM the strong color field inside the large cluster produces deceleration of the primary $q \bar{q}$ pair which can be seen as a thermal temperature. In case of A-A collisions it was observed that average transverse momentum is twice the temperature $\left\langle p_{t}\right\rangle=2 \mathrm{~T}$. This shows that the charged particle transverse momentum spectrum is exponentially distributed and the inverse slope parameter is the thermalized temperature [19].

After the initial temperature $T>T_{\mathcal{C}}$ the CSPM perfect fluid may expand according to Bjorken boost invariant 1D hydrodynamics [41]

$$
\varepsilon=\frac{3}{2} \frac{\frac{d N_{c}}{d y}\left\langle m_{t}\right\rangle}{S_{n} \tau_{\text {pro }}}
$$


where $\varepsilon$ is the energy density, $S_{n}$ nuclear overlap area, and $\tau_{\text {pro }}$ the production time for a boson (gluon) [25]. The $\tau_{\text {pro }}$ is given by Equation (2) $\sim 1 \mathrm{fm} / \mathrm{c}$. The dynamics of massless particle production has been studied in QE2 quantum electrodynamics. QE2 can be scaled from electrodynamics to quantum chromodynamics using the ratio of the coupling constants [25]. Figure 9 shows a plot of $\varepsilon / T^{4}$ as a function of $T / T_{\mathcal{C}}$ that is in excellent agreement with numerical lattice gauge calculations for $0.8<T / T_{\mathcal{C}}<2$. At $T / T_{\mathcal{C}} \sim 1.2(T \sim 195 \mathrm{MeV})$ we find that the $Q G P$ has $\sim 37$ quark gluon degrees of freedom.

It is important to ask the question if matter created in high multiplicity $p p$ events is nearly a perfect fluid with a low shear viscosity as observed in heavy ion collisions [19].

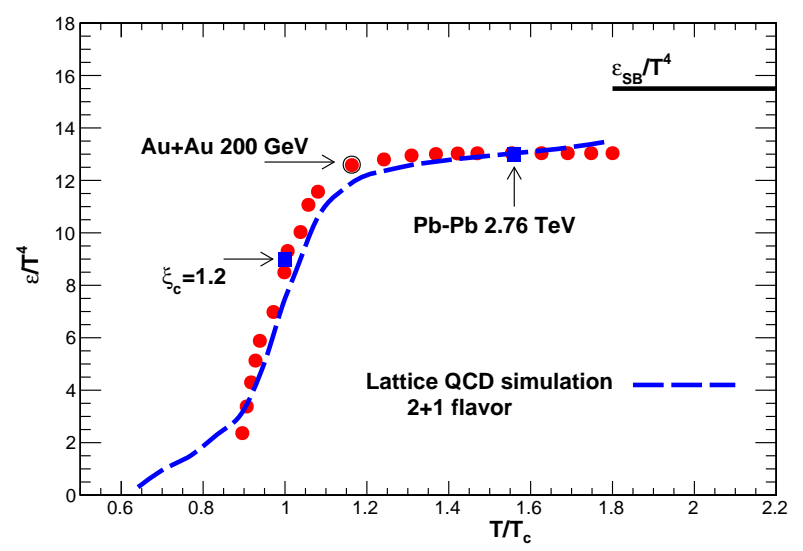

Figure 9. $\varepsilon / T^{4}$ versus $T / T_{\mathcal{C}}$ from CSPM (red circles) and Lattice QCD (blue dash line) for $2+1$ flavor and $\mathrm{p} 4$ action [20].

\section{Shear Viscosity and Trace Anomaly}

The shear viscosity to entropy density ratio $\eta / s$ was obtained in the framework of relativistic kinetic theory and the string percolation [42]. In this picture the relevant parameter is the string density $\xi$. The relativistic kinetic theory relation for the shear viscosity over entropy density ratio, $\eta / s$ is given by $[43,44]$

$$
\frac{\eta}{s} \simeq \frac{T \lambda_{m f p}}{5}
$$

where $T$ is the temperature and $\lambda_{m f p}$ is the mean free path given by

$$
\lambda_{m f p} \sim \frac{1}{\left(n \sigma_{t r}\right)}
$$

$n$ is the number density of an ideal gas of quarks and gluons and $\sigma_{t r}$ the transport cross section for these constituents.

After the cluster is formed it behaves like a free gas of constituents. Equation (22) can be applied to obtain the shear viscosity. In CSPM the number density is given by the effective number of sources per unit volume

$$
n=\frac{N_{\text {sources }}}{S_{N} L}
$$

where $L$ is the longitudinal extension of the source, $L=1.0-1.1 \mathrm{fm}$ [28]. $\eta / \mathrm{s}$ is obtained from Equations (22) and (24) as

$$
\frac{\eta}{S}=\frac{T L}{5\left(1-e^{-\tilde{\zeta})}\right.}
$$

Well below $\xi_{c}$, as the temperature increases, the string density increases and the area is filled rapidly and $\lambda_{m f p}$ and $\eta / s$ decrease sharply. Above $\xi_{c}$, more than $2 / 3$ of the area are already covered 
by strings, and therefore the area is not filling as fast and the relatively small decrease of $\lambda_{m f p}$ is compensated by the rising of temperature, resulting in a smooth increase of $\eta / s$. The behavior of $\eta / s$ is dominated by the fractional area covered by strings. This is not surprising because $\eta / s$ is the ability to transport momenta at large distances and that has to do with the density of voids in the matter.

Figure 10a shows $\eta / s$ as a function of the temperature. The lower bound shown in Figure 10a is given by the AdS/CFT conjecture [45]. The results from $p p$ collisions from $\sqrt{s}=13 \mathrm{TeV}$ shows a very small $\eta / s$ and that is 2.7 times the AdS/CFT conjectured lower bound $1 / 4 \pi$. The theoretical estimates of $\eta / s$ has been obtained as a function of $\mathrm{T} / T_{c}$ for both the weakly interacting (wQGP) and strongly interacting (sQGP) coupled QCD plasma are shown in Figure 5 [43].

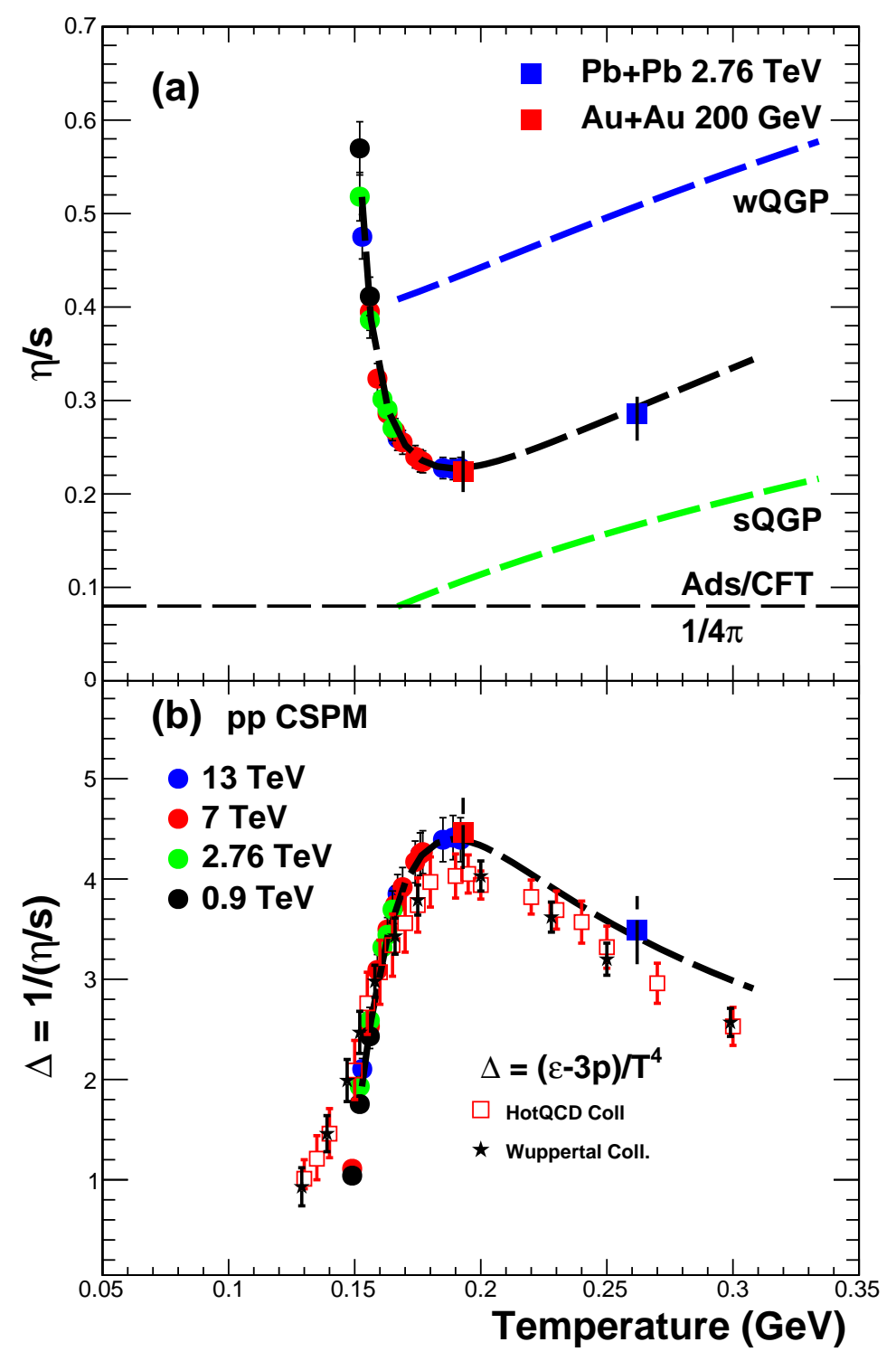

Figure 10. (a) $\eta / s$ as a function of temperature $\mathrm{T}$ using Equation (25) for $\sqrt{s}=0.9,2.76,7$ and $13 \mathrm{TeV}$. The lower bound shown is given by the AdS/CFT [45]. For comparison purposes the results from $\mathrm{Au}-\mathrm{Au}$ and $\mathrm{Pb}-\mathrm{Pb}$ at $\sqrt{s}_{N N}=200 \mathrm{GeV}$ and $2.76 \mathrm{TeV}$ respectively are also shown as solid squares for $0-5 \%$ centrality [19]. $\eta / s$ estimates for wQGP and sQGP are shown as dotted blue and green lines respectively [43]. (b) The trace anomaly $\Delta=(\varepsilon-3 p) / T^{4}$ vs. temperature. Blue open squares are from HotQCD Collaboration [46]. Black stars are from Wuppertal Collaboration [47]. The CSPM results are obtained as $\Delta=1 /(\eta / s)$ [19]. The black dashed line both in $(\mathbf{a}, \mathbf{b})$ corresponds to extrapolation from CSPM at higher temperatures. 
$\eta / s$ has also been obtained in several other calculations for pure glue matter [48], in the semi quark gluon plasma [49] and in quasi particle description [50]. In pure SU(3) gluodynamics a conservative upper bound for $\eta / s$ was obtained $\eta / s=0.134$ (33) at $T=1.65 T_{c}$ [51]. In the quasi particle approach also low $\eta / s \sim 0.2$ is obtained for $\mathrm{T}>1.05 \mathrm{~T}_{c}$ and rises very slowly with the increase in temperature [52]. In CSPM also $\eta / s$ grows with temperature as $0.16 T / T_{\mathcal{c}}$.

In CSPM $\eta / s$ is not needed to reproduce the elliptic flow and higher harmonics. The cluster formed by the strings has generally an asymmetric form in the transverse plane and acquires dimensions comparable to the nuclear overlap. This azimuthal asymmetry is at the origin of the elliptic flow in CSPM. The partons emitted at some point inside the cluster have to pass through the strong color field before appearing on the surface. The results of the simulation for different harmonics are in reasonable agreement with the experimental data on the $p_{t}$ and centrality dependencies [53,54].

The trace anomaly $(\Delta)$ is the expectation value of the trace of the energy-momentum tensor, $\left\langle\Theta_{\mu}^{\mu}\right\rangle=(\varepsilon-3 p)$, which measures the deviation from conformal behavior and thus identifies the interaction still present in the medium [55]. We consider the ansatz that inverse of $\eta / s$ is equal to trace anomaly $\Delta . \eta / s$ is in quantitative agreement with $(\varepsilon-3 p) / T^{4}$ over a wide range of temperatures [56,57]. Figure $10 \mathrm{~b}$ shows $1 /(\eta / s)$ and the dimensionless quantity, $(\varepsilon-3 p) / T^{4}$, obtained from lattice QCD simulations [46]. The minimum in $\eta / s \sim 0.22$ determines the peak of the interaction measure $\sim 4.5$ in agreement with the recent HotQCD values [46]. This happens at the temperature of $T_{\mathcal{C}} \sim 195 \mathrm{MeV}$. Figure 10b also shows the results from Wuppertal Collaboration [47]. The maximum in $\Delta$ corresponds to the minimum in $\eta / s$. Both $\Delta$ and $\eta / s$ describe the transition from a strongly coupled QGP to a weakly coupled QGP.

We are not aware of any theoretical work which directly relates the trace anomaly with the shear viscosity to entropy density ratio. However, the bulk viscosity $\zeta$ is related to both $\Delta$ and $\eta$ [58]. A detailed study based on low energy theorems and the lattice result for $\Delta$ shows that $\zeta / s$ rises very fast close to the critical temperature in such a way that its value at temperatures higher than $T>1.1 T_{\mathcal{C}}$ is quite negligible [59]. It was observed that $\zeta$ scales as $\alpha_{s}^{4} \eta$ where $\alpha_{s}$ is the coupling constant. The trace anomaly $\Delta$ is proportional to $\alpha_{s}^{2}$ [58]. There are many other works in which $\Delta$ and $\eta / s$ have been obtained separately $[48,50,60,61]$.

In our earlier work $F(\xi)$ was obtained in Au-Au collisions at $\sqrt{s}_{N N}=200 \mathrm{GeV}$ for various centralities using STAR data [19]. The results are shown in Figure 7 along with $p p$ (CMS) and $\bar{p} p$ (E735) collisions. It is observed that $F(\xi)$ as a function of $d N_{c} / \Delta \eta$ scaled by the transverse interaction area falls on a universal scaling curve for both hadron-hadron and nucleus-nucleus collisions. $F(\xi)$ values in high multiplicity events in $p p$ collisions at $\sqrt{s}=13 \mathrm{TeV}$ are similar to those obtained in most central events in Au-Au collisions at $\sqrt{s}_{N N}=200 \mathrm{GeV}$. In Figure 8 the temperatures from Au-Au collisions at $\sqrt{s}_{N N}=200 \mathrm{GeV}$ are compared with the $p p$ collisions. In high multiplicity events for $p p$ collisions at $\sqrt{s}=13 \mathrm{TeV}$ the temperature is the same as in most central events in Au-Au.

The $\eta / s$ as a function of temperature from $\mathrm{Au}-\mathrm{Au}$ at $\sqrt{s}_{N N}=200 \mathrm{GeV}$ and $\mathrm{Pb}-\mathrm{Pb}$ at $\sqrt{s}_{N N}=2.76 \mathrm{TeV}$ collisions is shown in Figure 10a along with $p p$ collisions. The $\eta / s$ value is similar both in the high multiplicity $p p$ collisions at $\sqrt{s}=13 \mathrm{TeV}$ and in most central collisions in Au-Au at $\sqrt{s}_{N N}=200 \mathrm{GeV}$. This shows that the QGP created in high multiplicity $p p$ collisions is also strongly coupled.

The similarity between high multiplicity $p p$ events and Au-Au suggests that the thermalization in both systems is reached through the stochastic process (Hawking-Unruh) instead of kinetic process [62].

\section{Summary and Conclusions}

In the initial collision of $p p$ and A-A nuclei at high energy, the Schwinger $Q E D_{2}$ mechanism scaled from electric to color fields, lifts color neutral $q \bar{q}$ pairs from the vacuum at the initial time $t_{i}=2.405$ $\bar{h} /<m_{t}>\sim 1 \mathrm{fm} / \mathrm{c}$. Here $<m_{t}>$ is the average transverse mass of quarks when a color source string breaks. Color sources are formed by the overlapping of $n$ individual color strings with transverse area $S_{1}$. The overall result of color string breaking is the increase of the transverse momentum $p_{t}$ of 
the $q \bar{q}$ pairs and the decrease of the multiplicity expected from $n$ individual strings. In the transverse plane the total color string area is reduced by $F(\xi)$ due to the overlapping of individual strings. $F(\xi)$ is determined by comparing the charged particle transverse momentum spectra from a lower energy $p p$ collision (single string) with the various high energy charged particle transverse momentum spectra from $p p$ and A-A collisions(overlapping strings). In the overlap area the strings start interacting to form clusters in the transverse plane very much similar to discs in 2D percolation theory. When the 2D percolation density parameter $\xi$ reaches $\xi=1.2$, the spanning cluster is formed and represents a communicating QGP droplet.

The initial temperature is given by Equation (16) and is due to the Hawking-Unruh barrier penetration of the event horizon provided by color confinement. The similarity between high multiplicity $p p$ events and $\mathrm{Au}-\mathrm{Au}$ indicates that the thermalization in both systems reached is due to a stochastic process rather than a kinetic process. We find $\sim 37$ degrees of freedom at highest temperature of $195 \mathrm{MeV}$ reached in $\mathrm{Au}-\mathrm{Au}$ collisions at $\sqrt{s_{N N}}=200 \mathrm{GeV}$.

The percolation clustering begins $\sim 145 \mathrm{MeV}$ and ends at the hadronization ie. critical temperature $(T=167.8 \mathrm{MeV})$. The center of this crossover phase transition is in agreement with the lattice gauge pseudo-critical temperature $T=155 \pm 9 \mathrm{MeV}$. The temperature dependence of the $\eta / s$ ratio across the crossover region has a clear minimum value at $T=195 \mathrm{MeV}$ and $\eta / s=0.22$, which is $\sim 2.7$ times the Ads/CFT value of $1 / 4 \pi$. Here the QGP has a low shear viscosity with strong residual interactions. We found that the inverse $\eta / s$ of closely tracks the lattice $Q C D$ values of the trace anomaly $\Delta=(\varepsilon-3 p) / T^{4}$ for $T$ from $150 \mathrm{MeV}$ to $260 \mathrm{MeV}$.

The color string percolation model (CSPM) gives a successful description of the experimental data in the soft region. The clustering of color sources is not derived directly from QCD, but has its most fundamental features. The non-Abelian character is reflected in the coherence sum of the color fields. Confinement is responsible for the event horizon and the small transverse size of the strings. The interpretation of data using color string percolation describes the equation of state of the QGP close to and above the critical (hadronization) temperature.

Author Contributions: All the authors contributed eqully to this work.

Funding: C.P. thanks the grant Maria de Maeztu Unit of excellence MDM-2016-0682 of Spain, and the support of Xunta de Galicia under the project ED431C 2017.

Conflicts of Interest: The authors declare no conflict of interest.

\section{References}

1. Alford, M.; Schmitt, A.; Rajagopal, K.; Schafer, T. Color superconductivity in dense dark matter. Rev. Mod. Phys. 2008, 80, 1455-1515. [CrossRef]

2. McLerran, L.; Redlich, K.; Sasaki, C. Quarkyonic matter and chiral symmetry breaking. Nucl. Phys. A 2009, 824, 86-100. [CrossRef]

3. Andronic, A.; Blaschke, D.; Braun-Munzinger, P.; Cleymans, J.; Fukushima, K.; McLerran, L.D.; Oeschler, H.; Pisarski, R.D.; Redlich, K.; Sasaki, C.; et al. Hadron production in ultra-relativistic nuclear collisions: Quarkyonic matter and a triple point in the phase diagram of QCD. Nucl. Phys. A 2010, 837, 65-86. [CrossRef]

4. Busza, W.; Rajagopal, K.; van der Schee, W. Heavy Ion Collisions: The Big picture, and the Big Questions. arXiv 2018, arXiv:1802.04801.

5. Nagle, J.; Zajc W. Small System Collectivity in Relativistic Hadronic and Nuclaer Collisions. arXiv 2018, arXiv:1801.03477.

6. Pasechnik, R.; Sumbera, M. Phenomenological Review of Quark-Gluon Plasma: Concepts and Observations. Universe 2017, 3, 7. [CrossRef]

7. Ratti, C. Lattice QCD and heavy ion collisions: a review of recent progress. arXiv 2018, arXiv:1804.07810.

8. Shen, C.; Heinz, U. The road to precision: Extraction of the specific shear viscosity of the QGP. Nucl. Phys. News 2015, 25, 6-11. [CrossRef]

9. Schwinger, J. Gauge variance and mass II. Phys. Rev. 1962, 128, 2425-2429. [CrossRef] 
10. Ferreres-Sole, S.; Sjostrand, T. The Space-Time Structure of Hadronization in the Lund Model. arXiv 2018, arXiv:1808.04619.

11. Satz, H. The analysis of dense matter. In Extreme States of Matter in Strong Interaction Physics: Lecture Notes in Physics; Springer: Berlin, Germany, 2012; Volume 841.

12. Braun, M.A.; Pajares, C. Implications of color-string percolation on multiplicities, correlations, and the transverse momentum. Eur. Phys. J. C 2000, 16, 349-359. [CrossRef]

13. Braun, M.A.; del Moral, F.; Pajares, C. Percolation of strings and the relativistic energy data on multiplicity and transverse momentum distributions. Phys. Rev. C 2002, 65, 024907. [CrossRef]

14. Satz, H. Color deconfinement in nuclear collisions. Rep. Prog. Phys. 2000, 63, 1511-1574. [CrossRef]

15. McLerran, L.; Venugopalan, R. Computing quark and gluon distribution functions for very large nuclei. Phys. Rev. D 1994, 49, 2233-2241. [CrossRef]

16. De Deus, J.D.; Pajares, C. String percolation and the Glasma. Phys. Lett. B 2001, 695, 211-213. [CrossRef]

17. Khachatryan, V.; Sirunyan, A. M.; Tumasyan, A.; Adam, W.; Bergauer, T.; Dragicevic, M.; Erö, J.; Fabjan, C.; Friedl, M.; Frühwirth, R.; et al. Observation of long range, near-side angular correlations in proton-proton collisions at the LHC. J. High Energy Phys. 2010, 9, 91. [CrossRef]

18. Khachatryan, V.; Sirunyan, A. M.; Tumasyan, A.; Adam, W.; Bergauer, T.; Dragicevic, M.; Erö, J.; Fabjan, C.; Friedl, M.; Frühwirth, R.; et al. Measurement of long range nearside two particle angular correlations in pp collisions at $\sqrt{s}=13$ TeV. Phys. Rev. Lett. 2016, 116, 172302. [CrossRef] [PubMed]

19. Braun, M.A.; de Deus, J.D.; Hirsch, A.S.; Pajares, C.; Scharenberg, R.P.; Srivastava, B.K. Deconfinement and clustering of color sources in nuclear collisions. Phys. Rep. 2015, 599, 1-50. [CrossRef]

20. Scharenberg, R.P.; Srivastava, B.K.; Hirsch, A.S. Percolation of color sources and the equation of state of QGP in central Au-Au collisions at $\sqrt{s_{N N}}=200 \mathrm{GeV}$. Eur. Phys. J. C 2011, 71, 1510. [CrossRef]

21. Tsallis, C. Possible generalization of Boltzmann-Gibbs statistics. J. Stat. Phys. 1988, 52, 479-487. [CrossRef]

22. McLerran, L.; Praszalowicz, M.; Schenke, B. Transverse momentum of protons, pions and kaons in high multiplicity $p p$ and $p A$ collisions; evidence of the Color Glass Condensate? Nucl. Phys. A 2013, 916, 210-218. [CrossRef]

23. Loizides, C. Glauber modeling of high-energy nuclear collisions at the subnucleon level. Phys. Rev. C 2016, 94, 024914. [CrossRef]

24. Gutay, L.G.; Hirsch, A.S.; Pajares, C.; Scharenberg, R.P.; Srivastava, B.K. Deconfinement in small systems: Clustering of color sources in high multiplicity $\bar{p} p$ collisions at $\sqrt{s}=1.8 \mathrm{TeV}$. Int. J. Mod. Phys. E 2015, 24, 155010. [CrossRef]

25. Wong, C.Y. Introduction to High Energy Heavy Ion Collisions; World Scientific: Singapore, 1994; p. 289.

26. Bialas, A. Fluctuations of the string tension and transverse mass distribution. Phys. Lett. B 1999, 466, 301-304. [CrossRef]

27. Dosch, H.G. Gluon condensate and effective linear potential. Phys. Lett. B 1987, 190, 177-181. [CrossRef]

28. Dias de Deus, J.; Pajares, C. Percolation of color sources and critical temperature. Phys. Lett. B 2006, 642, 455-458. [CrossRef]

29. Braun-Munzinger, P.; Stachel, J.; Wetterich, C. Chemical freeze-out and the QCD phase transition temperature. Phys. Lett. B 2004, 596, 61-69. [CrossRef]

30. Becattini, F.; Castorina, P.; Milov, A.; Satz, H. A comparative analysis of statistical hadron production. Eur. Phys. J. C 2010, 66, 377-386. [CrossRef]

31. Kharzeev, D.; Levin, E.; Tuchin, K. Multiparticle production and thermalization in high-enrgy QCD. Phys. Rev. C 2005, 75, 044903. [CrossRef]

32. Hawking, S.W. Particle creation by black holes. Commun. Math. Phys. 1975, 43, 199-220. [CrossRef]

33. Unruh, W.G. Notes on black-hole evaporation. Phys. Rev. D 1976, 14, 870-892. [CrossRef]

34. Castorina, P.; Kharzeev, D.; Satz, H. Thermal hadronization and Hawking-Unruh radiation in QCD. Eur. Phys. J. C 2007, 52, 187. [CrossRef]

35. Bylinkin, A.A.; Kharzeev, D.E.; Rostovtsev, A.A. The origin of thermal componenet in the transverse momentum spectra in high energy hadronic processes. arXiv 2014, arXiv:1407.4087.

36. Castorina, P.; Satz, H. Hawking-Unruh hadronization and strangeness production in high energy collisions. Adv. High Energy Phys. 2014, 2014, 376982. [CrossRef] 
37. Adcox, K.; Adler, S.S.; Afanasiev, S.; Aidala, C.; Ajitanand, N.N.; Akiba, Y.; Al-Jamel, A.; Alexander, J.; Amirikas, R.; Aoki, K.; et al. Formation of dense partonic matter in relativistic nucleus-nucleus collisions at RHIC: Experimental evaluation by the PHENIX Collaboration. Nucl. Phys. A 2005, 757, 184-283. [CrossRef]

38. Muller, B.; Schukraft, J.; Wyslouch, W. First results from Pb-Pb collisions at the LHC. Ann. Rev. Nucl. Part Sci. 2012, 62, 361-386. [CrossRef]

39. Adam, J.; Adamova, D.; Aggarwal, D.D.; Agalieri Rinella, G.; Agnello, M.; Agrawal, N.; Ahammed, Z.; Ahn, S.U.; Aiola, S.; Akindinov, A.; et al. Direct photon production in Pb-Pb collisions at $\sqrt{s_{N N}}=2.76 \mathrm{TeV}$. Phys. Lett. B 2016, 754, 235-248. [CrossRef]

40. Becattini, F.; Steinheimer, J.; Stock, R.; Bleicher, M. Hardonization conditions in relativistic nuclear collisions and the QCD pseudo-critical line. Phys. Lett. B 2017, 764, 241-246. [CrossRef]

41. Bjorken, J.D. Highly relativistic nucleus-nucleus collisions: The central rapidity region. Phys. Rev. D 1983, 27, 140. [CrossRef]

42. Dias de Deus, J.; Hirsch, A.S.; Pajares, C.; Scharenberg, R.P.; Srivastava, B.K. Clustering of color sources and the shear viscosity of the QGP in heavy ion collisions at RHIC and LHC energies. Eur. Phys. J. C 2012, 72, 2123. [CrossRef]

43. Hirano, T.; Gyulassy, M. Perfect fluidity of the quark-gluon plasma core as seen through its dissipative hadronic corona. Nucl. Phys. A 2006, 769, 71-94. [CrossRef]

44. Danielewicz, P.; Gyulassy, M. Dissipative phenomena in quark-gluon plasma. Phys. Rev. D 1985, $31,53$. [CrossRef]

45. Kovtun, P.K.; Son, D.T.; Starinets, A.O. Viscosity in strongly interacting quantum field theories from black hole physics. Phys. Rev. Lett. 2005, 94, 111601. [CrossRef] [PubMed]

46. Bazavov, A.; Bhattacharya, T.; DeTar, C.; Ding, H.T.; Gottlieb, S.; Gupta, R.; Hegde, P.; Heller, U.M.; Karsch, F.; Laermann, E.; et al. Equation of state in (2+1)-flavor QCD. Phys. Rev. D 2014, 90, 094503. [CrossRef]

47. Borsányi, S.; Fodor, Z.; Hoelbling, C.; Katz, S.D.; Krieg, S.; Szabo, K.K. Full result for the QCD equation of state with $2+1$ flavors. Phys. Lett. B 2014, 730, 99-104. [CrossRef]

48. Khvorostukhin, A.S.; Toneev, V.D.; Voskresensky, D.N. Shear and bulk Viscosities of pure glue matter. Phys. Rev. C 2011, 83, 035204. [CrossRef]

49. Hidaka, Y.; Pisarski, R.D. Small shear viscosity in the semiquark gluon plasma. Phys. Rev. D 2010, 81, 076002. [CrossRef]

50. Bluhm, M.; Kampfer, B.; Redlich, K. Bulk and shear viscosities of the gluon plasma in quasiparticle description. Phys. Rev. C 2011, 84, 025201. [CrossRef]

51. Meyer, H.B. Calculation of shear viscosity in SU(3) gluodynamics. Phys. Rev. D 2007, 76, 101701. [CrossRef]

52. Peshier, A.; Cassing, W. The hot nonperturbative gluon plasma is an almost colored liquid. Phys. Rev. Lett. 2005, 94, 172301. [CrossRef] [PubMed]

53. Bautista, I.; Dias de Deus, J.; Pajares, C. Elliptic flow at RHIC and LHC in the string percolation approach. Eur. Phys. J. C 2012, 72, 2038. [CrossRef]

54. Braun, M.A.; Pajares, C.; Vechernin, V.V. Anisotropic flow from color strings: Monte-Carlo simulations. Nucl. Phys. A 2013, 906, 14-27. [CrossRef]

55. Cheng, M.; Ejiri, S.; Hegde, P.; Karsch, F.; Kaczmarek, O.; Laermann, E.; Mawhinney, R.D.; Miao, C.; Mukherjee, S.; Petreczky, P.; et al. Equation of state for physical quark masses. Phys. Rev. D 2010, 81, 054510. [CrossRef]

56. Scharenberg, R.P. The QGP equation of state by measuring the color suppression factor at RHIC and LHC energies. In Proceedings of the 8th International Workshop on Critical Point and Onset of Deconfinement, Napa, CA, USA, 11-15 March 2013.

57. Srivastava, B.K. Percolation approach to initial stage effects in high energy collisions. Nucl. Phys. A 2014, 926, 142-151. [CrossRef]

58. Schafer, T.; Teaney, D. Nearly perfect fluidity: From cold atomic gases to hot quark gluon plasma. Rep. Prog. Phys. 2009, 72, 126001. [CrossRef]

59. Karsch, F.; Kharzeev, D.; Tuchin, K. Universal properties of bulk viscosity near the QCD phase transition. Phys. Lett. B 2008, 663, 217-221. [CrossRef]

60. Plumari, S.; Alberico, W.M.; Greco, V.; Ratti, C. Recent thermodynamic results from lattice QCD analyzed within a quasiparticle model. Phys. Rev. D 2011, 84, 094004. [CrossRef] 
61. Marty, R.; Bratkovskaya, E.; Cassing, W.; Aichelin, J.; Berrehrah, H. Transport coefficients from the Nambu-Jona-Lasinio model for SU(3) $)_{f}$. Phys. Rev. C 2013, 88, 045204.

62. Satz, H. Extreme states of matter in strong interaction physics. Lect. Notes Phys. 2012, 841, 52-56.

(C) 2018 by the authors. Licensee MDPI, Basel, Switzerland. This article is an open access article distributed under the terms and conditions of the Creative Commons Attribution (CC BY) license (http:/ / creativecommons.org/licenses/by/4.0/). 